\title{
Linewidth-modulated motional Stark effect measurements of internal field structure in low-field configurations
}

\author{
E. A. Reinecke, ${ }^{\text {a) }}$ R. J. Fonck, ${ }^{\text {b) }}$ and T. A. Thorson \\ University of Wisconsin-Madison, 1500 Engineering Drive, Madison, Wisconsin 53706
}

(Presented on 21 June 2000)

\begin{abstract}
Motional Stark effect measurements of internal field structure in low-field magnetic confinement configurations are considered for both magnitude and direction of the local magnetic field. The amplitude and phase delay of an oscillating spectral linewidth driven by a rotating polarizer provides a means of determining the magnitude and direction of the total field simultaneously while avoiding difficulties of neutral beam energy drift. Photon-noise limit estimates for a diagnostic beam on the low-field Pegasus toroidal experiment indicate sensitivities of roughly $20 \mathrm{G}$ and $0.2^{\circ}$ for the magnitude and direction angle. These values are sufficient to provide significant constraints on magnetic equilibrium reconstructions. (C) 2001 American Institute of Physics.
\end{abstract}

[DOI: $10.1063 / 1.1319605]$

\section{INTRODUCTION}

The equilibrium state in a toroidal magnetically confined plasma is determined by the poloidal flux functions of pressure $P(\psi)$ and poloidal current $G(\psi)$ across the magnetic flux surfaces. To understand the equilibrium and stability properties of such plasmas, it is desirable to constrain the allowed values of $P(\psi)$ and $G(\psi)$ through measurements of the internal magnetic field structure. This is especially true in the present generation of alternate magnetic confinement configurations such as the spherical torus $(\mathrm{ST}),{ }^{1}$ the spheromak, and the reversed field pinch.

Both collisionally induced fluorescence and laserinduced fluorescence approaches are under development to extend motional Stark effect (MSE) measurements to lowfield devices $(0.1-0.3 \mathrm{~T}){ }^{2-4}$ We consider here an evaluation of collisionally induced MSE measurements on the PEGASUS toroidal experiment ${ }^{5}$ for determination of the plasma equilibrium and stability properties at very high toroidal betas. PEgASUS is an ultralow aspect ratio ST with $R \sim 0.45 \mathrm{~m}, a$ $\sim 0.4 \mathrm{~m}, I_{p} \sim 0.3 \mathrm{MA}$, and $B_{t}($ vacuum $)<0.1 \mathrm{~T}$.

Two features distinguish the present study: (1) the assumed extremely low $B_{t}$; and (2) the requirement that both the tilt of the field lines and the total magnetic field strength be measured. The field line tilt angle gives a strong constraint on the plasma current density $j(\psi)$ and hence $G(\psi)$, while the total field constrains the plasma pressure $P(\psi)$.

In this article, we first consider the Stark structure from a model diagnostic neutral beam injected into the PEGASUS plasma. Attention is given to those issues that are especially challenging at low field. We then discuss MSE measurements via a linewidth modulation technique as an approach which can provide sufficient sensitivity for PEGASUS-like conditions. This is followed by signal-to-noise estimates for a practical beam and spectrometer arrangement.

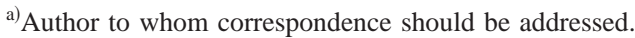

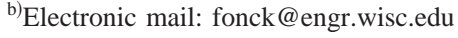

\section{MODEL MOTIONAL STARK SPECTRA}

The motional Stark effect arises from the electric field induced in a neutral beam atom's rest frame as it crosses the internal magnetic field of the plasma, $\mathbf{E}=\boldsymbol{\nu} \times \mathbf{B}$. Hydrogen atoms experience a linear Stark splitting, with the $\mathrm{H}_{\alpha}(3-2)$ transition separating into nine components of significant intensity, but this spectrum usually appears as a triplet structure due to several broadening influences. The energy difference between the Stark manifold components depends on the total electric field perpendicular to the optical line of sight, with the central lines being polarized perpendicular $(\sigma)$ to the direction of the electric field and the symmetric satellite lines polarized parallel $(\pi)$ to it. Ideally, one wants to measure the direction of polarization to obtain the field line tilt angle and the magnitude of the line splitting to measure the total magnetic field.

The problems inherent in these measurements at low field are well understood, as the Stark splitting is small due to the low fields in the plasma. ${ }^{3,4}$ Several processes conspire to produce significant line broadening of the observed spectrum, making it difficult to resolve the line structure and/or measure small fractional polarization directions. Line broadening arises from the divergence of velocities in neutral beam sources, thermal broadening of the beam neutrals, limited resolution of the requisite high-throughput spectrometer, and line-of-sight broadening. The last arises from the range of viewing angles which, with the beam emissions, are sampled across an optical collection system. This is, in practice, the limiting factor for achievable resolution at low fields. On PEGASUs, the line-of-sight broadening is reduced by limiting the width of the collection optics to values where the Stark spectrum is partially resolved. Since the Stark splitting is on the order of $1-2 \AA$, the lens width is practically limited to $\sim 1 \mathrm{~cm}$ for the lens-beam distance of $\sim 60 \mathrm{~cm}$. These limitations are exacerbated by the relatively low beam energies in the diagnostic neutral beams often available to small facilities. The net result is relatively low polarization fractions and poorly resolved Stark line structure. ${ }^{4}$ 

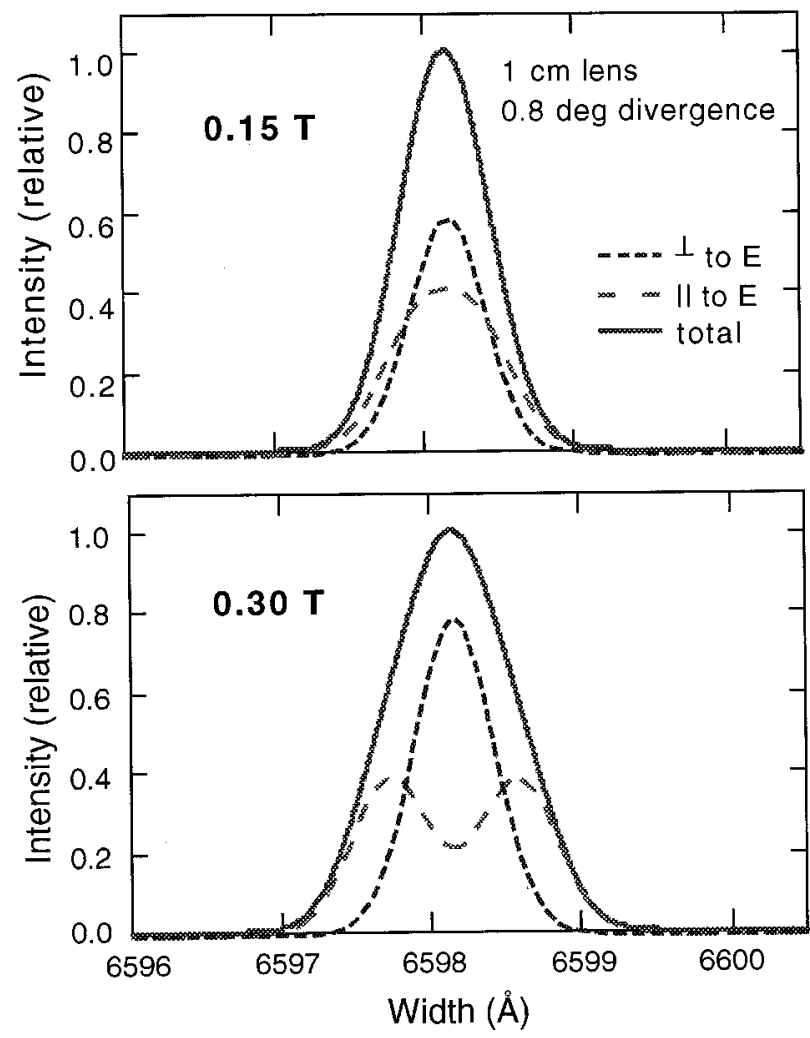

FIG. 1. Calculated Stark line profiles for $\mathrm{H}_{\alpha}$ at representative magnetic fields for the PEGASUs viewing geometry.

A final critical complication is the Stark contributions from the intrinsic radial electric field in the plasma. ${ }^{3}$ At low magnetic fields, this intrinsic radial field can become comparable to or larger than the induced motional field and make MSE measurements extremely problematic at best. Therefore, on PEgasus, we require a strictly radial line-of-sight when collecting light from the beam. This in turn requires that the beam injection direction be variable to provide spatial profiles.

With these conditions, model spectra have been calculated for a $30 \mathrm{keV} \mathrm{H}^{0}$ neutral beam for a realistic viewing geometry in PEgasus. The model includes the basic Stark manifold, the beam thermal broadening $(1 \mathrm{eV})$, the divergence broadening $\left(0.8^{\circ}\right)$, and the line-of-sight broadening for a $1 \mathrm{~cm}$ lens and $0.5 \mathrm{~cm}$ wide sample area in the beam cross section. Figure 1 shows sample spectra calculated for $B_{\text {total }}=0.15 \mathrm{~T}$ and $0.3 \mathrm{~T}$, the range of values expected for highly paramagnetic plasmas with vacuum fields $B_{\text {vac }}$ $\sim 0.05-0.15 \mathrm{~T}$. The Stark structure is only barely resolved at the higher field strength and manifested at the low field by an apparent polarization-dependent linewidth. Nevertheless, the influence of the motional Stark field on the basic line structure is readily evident. To see this, Fig. 2 shows the full width at half maximum (FWHM) of the $\pi$ and $\sigma$ components individually and the total linewidth as functions of total magnetic field.

\section{LINEWIDTH MODULATION APPROACH}

The goal here is to measure both the direction of the magnetic field line, which is given by the direction of polar-

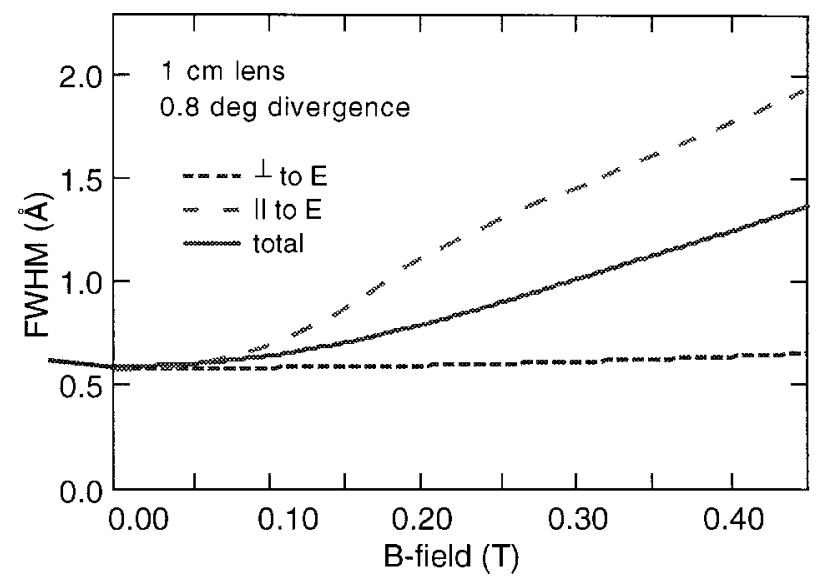

FIG. 2. FWHM vs total magnetic field for the total line and the separate polarized components.

ization of the components of the Stark manifold, and the total magnetic field strength, which in turn is given by the magnitude of the Stark splitting or broadening (in the unresolved case). This is achieved by comparison of measurements of the $\pi$ and $\sigma$ components of the line structure.

Such measurements can, in principle, be achieved by detailed line-structure measurements with a high-resolution spectrometer and multichannel charge coupled device detector, wherein one measures two spectra at different polarization angles. An alternative approach, which we consider here, is to use linewidth modulation spectroscopy and borrow techniques from turbulence measurements. ${ }^{6-9}$ The basic approach is to rotate a linear polarizer at frequency $\Omega$ to repeatedly vary the observed spectrum from the $\pi$ to $\sigma$ components, and use a high-speed, high-throughput multichannel spectrometer to measure the oscillations in the resultant linewidth. This linewidth will modulate at a fundamental frequency of $2 \Omega$ and the frequency characteristics of this linewidth are readily measured as the frequency characteristics of the second moment of the spectral distribution

$$
V \equiv \frac{\int I(\lambda)[\lambda-\bar{\lambda}]^{2} d \lambda}{\int I(\lambda) d \lambda} \cong \frac{\sum_{j=1}^{M} I\left(\lambda_{j}\right)\left[\lambda_{j}-\bar{\lambda}\right]^{2}}{\sum_{j=1}^{M} I\left(\lambda_{j}\right)} .
$$

As shown in the earlier studies of ion temperature turbulence, ${ }^{8}$ the photon-statistics noise level in a measure of the variance $V$ is related directly to the total number of photons detected, $N_{\gamma}$. In particular, the noise power density in $V$ is given by the inverse of the total photon count rate across the spectral line:

$$
\frac{\sigma_{v}}{V} \cong \sqrt{\frac{4 \mathrm{BW}}{d N_{\gamma} / d t}},
$$

where $\mathrm{BW}$ is the frequency bandwidth over which the signal is integrated.

The total field strength is obtained from the amplitude of the fluctuating linewidth, while the phase difference between the observed linewidth fluctuation and the polarizer angle gives the field line tilt angle. In both cases, the extraction of the desired measurable is aided by the fact that one is performing cross-spectrum analysis with a clean reference signal. This minimizes the noise floor in each measurement. 
The actual statistical noise floor is raised by the finite spectrometer resolution which reduces the modulation depth of the linewidth. ${ }^{8}$ The photon-noise level in the actual Stark manifold variance is given by the product of the measured linewidth and the logarithmic derivative of the real and measured linewidths

$$
\frac{\widetilde{V}_{\text {real }}}{V_{\text {real }}}=\frac{d \ln \left(V_{\text {real }}\right)}{d \ln \left(V_{\text {meas }}\right)} \frac{\widetilde{V}_{\text {meas }}}{V_{\text {meas }}} .
$$

The logarithmic scale factor is readily calculated by modeling the line structure with the known spectrometer passband. ${ }^{8}$ It plays the same role as a reduction in the polarization fraction does in conventional MSE measurements in that it reduces the contrast of the desired measurement.

Measurement of the linewidth modulation has the advantage that it is not susceptible to variations in the beam energy since the measurement analysis automatically compensates for changes in the lower order moments of the linewidth (i.e., the total intensity and line center or $E_{B}$ ). A time-averaged measure of the total field is readily produced.

Compared to conventional MSE polarization measurements, which use a single spectral channel for polarization measurements, this approach provides a modest decrease in the photon statistics noise floor since all photons across the spectral line are used. In practice, this is at most about a factor of two improvement. More importantly, the linewidth modulation approach offers a path to the total field measurement.

The main disadvantage compared to conventional MSE is the need for more a complex multichannel spectrometer assembly, with its attendant calibration requirements. This in turn makes the expansion to multiple spatial channels more costly. This may be alleviated by use of more advanced spectrometer concepts. ${ }^{10}$ In any case, it may be a worthwhile approach for experiments such as PEGASUs where even a single spatial point measurement can provide a significant added constraint on the magnetic equilibrium reconstruction.

\section{SENSITIVITY ESTIMATES}

We consider here a model spectrometer design to estimate the expected signal-to-noise values of these measurements for PEgASUs-like conditions. We assume that the minimal linewidth amplitude and cross-phase measurements are limited by photon statistical noise to derive the noise floor. Of course, additional systematic uncertainties will raise the total uncertainty, but photon noise level estimates can provide sufficient information to guide deployment decisions.

The baseline spectrometer design is that used for the original HF-CHERS ion temperature fluctuation experiment. ${ }^{8}$ It consists of an $f / 4$ Echelle grating spectrometer with a $1 / 2 \mathrm{~m}$ focal length. With a resolution of $\sim 0.4 \AA$ (0.1 mm slits) and $2 \mathrm{~cm}$ high slits, the optical throughput is $\sim 0.001 \mathrm{~cm}^{2}$ sr. This matches a collection lens of $6 \mathrm{~cm}$ high and $1 \mathrm{~cm}$ wide with a sample area in the plasma of $2 \mathrm{~cm}$ high by $0.5 \mathrm{~cm}$ wide. The rectangular lens is used to minimize the line-of-sight broadening in a manner similar to that employed on the National Spherical Torus Experiment. ${ }^{11}$ The

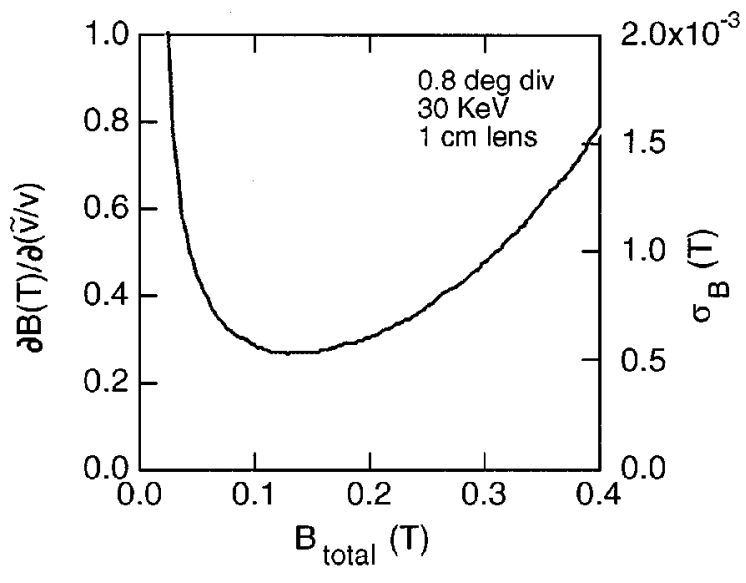

FIG. 3. (Left) Inverse derivative of linewidth amplitude vs total magnetic field. (Right) Photon-noise uncertainty level in total field from fluctuation amplitude measurement.

throughput of this system can readily be increased by a factor of 2 or more by employing multiple viewing lenses and shifted entrance slit assemblies, if needed.

The neutral beam is assumed to be a small diagnostic beam with $E_{B}=30 \mathrm{keV}, I_{B}=1.5 \mathrm{~A}$, and a net attenuation in the plasma core region of about 0.3 . The population of the $n=3$ excited state of $\mathrm{H}^{0}$ is derived from a multistate beam attenuation/excitation model to be 0.0012 , giving a total line intensity of $2 \times 10^{12}$ photons $/ \mathrm{cm}^{2} \mathrm{~s} \mathrm{sr}$.

We assume an avalanche photodiode detector array with an effective quantum efficiency (after accounting for the excess noise factor) of 0.4 , plus a net spectrometer and collection optics efficiency of $0.1-0.2$. Over a $5 \mathrm{~ms}$ integration time, this gives the total number of photons collected across the line profile to be $N_{\gamma} \sim 10^{6}$.

The minimal uncertainty in the total magnetic field is given by the statistical noise in the linewidth fluctuation amplitude in the limiting sample bandwidth, and is

$$
\sigma_{B} \approx\left[\frac{\partial B}{\partial(\widetilde{V} / V)}\right]\left[\frac{\partial\left(\ln V_{\text {real }}\right)}{\partial\left(\ln V_{\text {meas }}\right)}\right] \frac{\sigma_{v}}{V},
$$

where the first term is derived from the Stark line model, the second is the aforementioned contribution from finite instrumentation linewidth ( $\sim 2$ for the baseline model), and the last term is derived from the minimal uncertainty in the variance of the intensity distribution $\left(N_{\gamma}^{-1 / 2} \sim 10^{-3}\right)$. The resulting minimal noise in the total field measurements is shown in Fig. 3, along with the derivative of the magnetic field with respect to the relative linewidth from the Stark line model. Even if the final noise floor is $2-3$ times greater than these photon-noise floor estimates, the uncertainty in $B$ is on the order of $20 \mathrm{G}$ or so. Measurements of $B$ with this uncertainty level should provide strong constraints on the magnetic equilibrium reconstruction.

Uncertainty estimates of the field line tilt angle (i.e., $\left.\tan ^{-1}\left[B_{\mathrm{pol}} / B_{\text {tor }}\right]\right)$ are derived in a similar manner by considering the statistics-limited uncertainty in the cross phase between the linewidth fluctuation measurement and the reference polarization angle. The minimal error in the cross phase 


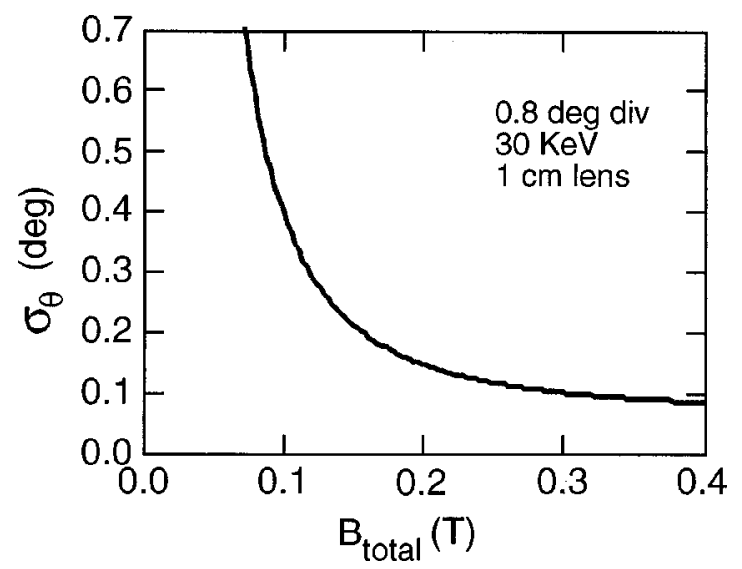

FIG. 4. Statistical uncertainty in measured tilt angle vs total magnetic field.

angle at frequency $f$ depends on the coherence function between the linewidth and polarization angle waveforms. ${ }^{12}$

For the case where the measured line variance is given by a harmonic oscillation with a constant phase shift plus a small amount of noise, the coherence $\left(\gamma^{2}\right)$ can be shown to depend on the ratio of the spectral power density in the photon noise to that in the modulating linewidth

$$
\begin{aligned}
& \gamma_{m, P}^{2}(f) \approx \frac{1}{1+\frac{d P_{n}}{d f} / \frac{d P_{\nu}}{d f}} \approx \frac{1}{1+2 /\left[\sqrt{N}\left(\widetilde{\nu}_{m} / \nu_{m}\right)^{2}\right]}, \\
& \sigma_{\theta} \approx \frac{1}{\sqrt{N}\left(\widetilde{\nu}_{m} / \nu_{m}\right)} .
\end{aligned}
$$

Equation 5(b) gives the statistics-limited uncertainty estimate in the cross phase. This quantity is shown in Fig. 4 as a function of total field for the reference case depicted in Fig. 1. The photon-noise limit to the tilt angle is then of order $0.2^{\circ}$, which is comparable to that obtained on the high-field tokamak plasmas. It corresponds to an uncertainty in the central magnetohydrodynamics (MHD) safety factor of $q(0) \sim \pm 0.04$ for PEgasus-like plasmas. Even with added systematic uncertainties, the levels for $\sigma_{\theta}$ are small enough to constrain the current density profile in the MHD reconstruction. This is especially true for larger radii, where the field line tilt is quite large as $B_{\mathrm{pol}}>B_{\text {tor }}$ and $\theta>45^{\circ}$.

\section{SUMMARY}

Variations on the design discussed above could be employed to improve the projected signal-to-noise ratios for the field magnitude and tilt angle by a factor of 2 or more. Higher throughput spectrometers, multislit spectrometers, multilens collection optics, and a higher energy diagnostic beam would all increase the capabilities of such measurements. Even so, the baseline design suggests that the linewidth modulation measurements discussed here have the potential to provide significant internal field measurements (both magnitude and orientation) in low-field configurations such as the ST.

\section{ACKNOWLEDGMENTS}

The authors wish to gratefully acknowledge helpful discussions with F. Levinton, B. Stratton, and K. Burrell. This work was supported by the U.S. Department of Energy Grant No. DE-FG02-96ER54375.

${ }^{1}$ A. W. Morris et al., Plasma Phys. Controlled Fusion 41, B191 (1999).

${ }^{2}$ F. M. Levinton, G. M. Gammel, H. W. Kugel, and D. W. Roberts, Rev. Sci. Instrum. 61, 2914 (1990).

${ }^{3}$ B. W. Rice, D. G. Nilson, K. H. Burrell, and L. L. Lao, Rev. Sci. Instrum. 70, 815 (1999).

${ }^{4}$ F. M. Levinton, Rev. Sci. Instrum. 70, 810 (1999).

${ }^{5}$ R. J. Fonck et al., Bull. Am. Phys. Soc. 43, 1867 (1998).

${ }^{6}$ R. J. Fonck, P. A. Duperrex, and S. F. Paul, Rev. Sci. Instrum. 61, 3487 (1990).

${ }^{7}$ H. T. Evensen, R. Durst, R. J. Fonck, and S. F. Paul, Rev. Sci. Instrum. 66, 845 (1995).

${ }^{8}$ H. T. Evensen, R. J. Fonck, S. F. Paul, G. Rewoldt, S. D. Scott, W. M. Tang, and M. C. Zarnstorff, Nucl. Fusion 38, 237 (1998).

${ }^{9}$ D. J. Den Hartog, A. F. Almagri, S. C. Prager, and R. J. Fonck, Rev. Sci. Instrum. 66, 444 (1995)

${ }^{10} \mathrm{~J}$. Howard et al., Rev. Sci. Instrum. (these proceedings).

${ }^{11}$ F. M. Levinton (private communication, 2000).

${ }^{12}$ J. S. Bendat and A. G. Piersol, Random Data Analysis and Measurement Procedures, 2nd ed. (Wiley, New York, 1986), p. 317. 